\title{
Does Plasmodium Infection Affect Mosquito Attraction?
}

\author{
Diego Santiago-Alarcon ${ }^{1 *}$ and Francisco C. Ferreira ${ }^{2,3 *}$ \\ ${ }^{1}$ Red de Biología y Conservación de Vertebrados, Instituto de Ecología, Xalapa, Mexico, ${ }^{2}$ Center for Conservation \\ Genomics, Smithsonian Conservation Biology Institute, Washington, DC, United States, ${ }^{3}$ Center for Vector Biology, Rutgers \\ University, New Brunswick, NJ, United States
}

OPEN ACCESS

Edited by:

Laura Gangoso,

Consejo Superior de Investigaciones

Cientificas (CSIC), Spain

Reviewed by:

Rafael Gutiérrez López,

University of Porto, Portugal

Leanne Grieves,

McMaster University, Canada

Jiayue Yan

University of Illinois

at Urbana-Champaign, United States

*Correspondence:

Diego Santiago-Alarcon diego.santiago@inecol.mx;

onca77@gmail.com

Francisco C. Ferreira

franciscocarlosfj@gmail.com

Specialty section:

This article was submitted to

Behavioral and Evolutionary Ecology,

a section of the journal

Frontiers in Ecology and Evolution

Received: 13 July 2020

Accepted: 21 October 2020

Published: 09 November 2020

Citation:

Santiago-Alarcon D and Ferreira FC (2020) Does Plasmodium Infection Affect Mosquito Attraction?

Front. Ecol. Evol. 8:582943.

doi: $10.3389 /$ fevo.2020.582943
Vector-host interactions play a central role in the transmission of vector-borne diseases. Determining the factors that affect vector attraction to vertebrate hosts is critical to understand disease ecological dynamics. Using malaria as the primary model, we reviewed studies that examined whether pathogen infection and host traits affect mosquito attraction. We found contradictory results examining mosquito attraction to birds infected with Plasmodium parasites, presumably because of widely variable experimental approaches. We summarize current experimental findings and propose standardized experimental approaches for future studies.

Keywords: behavioral manipulation, vector attraction, experimental parasitology, arboviruses, avian malaria, Culicidae, Diptera

\section{INTRODUCTION}

Insect vectors have often been considered passive vessels that carry pathogens without exerting much selection. However, vectors may suffer high pathogen-related mortalities (Valkiūnas et al., 2014; Gutiérrez-López et al., 2019a,b), and vectors selection of vertebrate hosts to obtain blood meals is not random or solely based on host availability, they follow general and specific host cues such as carbon dioxide and volatile body chemicals (Takken and Verhulst, 2013). Interestingly, both host choice and host attractiveness may be manipulated by the parasite in order to increase its transmission success (i.e., manipulation hypothesis; Heil, 2016).

The process by which pathogens manipulate vectors' behavior can be indirect during the development in a vertebrate host (e.g., increased attractiveness of infected hosts to uninfected vectors), and/or direct during the cycle inside the vectors (e.g., increase in attraction or feeding rates toward uninfected hosts; Gandon, 2018). These changes have profound implications for pathogen transmission. For instance, uninfected vectors highly attracted toward infected hosts would initially increase transmission in local populations (Gandon, 2018). Generalist mosquito species feeding on birds and mammals (depending on host availability; Abella-Medrano et al., 2018) play important roles in the transmission of zoonotic pathogens (e.g., Culex pipiens transmitting West Nile virus (WNV) and Usutu (USUV) virus among birds, equids, and humans; Brugman et al., 2018; Rochlin et al., 2019). Thus, investigating drivers of mosquito host selection is essential to understand the dynamics of vertebrate-vector-pathogen systems.

In recent years avian haemosporidian parasites have received attention as model systems to understand the ecology and evolution of vector-borne parasites (Rivero and Gandon, 2018). In particular because they are widespread, infect a large proportion of bird species worldwide, and affect bird fitness and survival (Atkinson and Van Riper, 1991; Valkiūnas, 2005; 
Palinauskas et al., 2020). Thus, by focusing on avian malaria, here we provide an overview of the factors affecting host selection by mosquitoes concentrating on Culex mosquitoes, which transmit human pathogens, bird pathogens, and zoonotic pathogens that use birds as reservoirs (WNV, USUV). Although Culex mosquitoes have a broad range of hosts, some are predominantly mammophilic and others are ornithophilic (e.g., Culex theileri vs. Cx. pipiens), yet their feeding behaviors depend on the species and population of origin, which also affects the parasites they transmit (Santa-Ana et al., 2006; Farajollahi et al., 2011; Rochlin et al., 2019).

Birds are used as models to analyze factors within species that affect host attractiveness to mosquitoes, which include mosquito and bird pathogen infection status, differences in the production of natural chemicals by birds (uropygial gland secretion and volatile compounds), host sex, and interactions among some of these factors (Table 1). We took advantage of studies on human and mice malaria systems that also addressed some of these factors to suggest next steps for studies on bird attractiveness to mosquitoes. We found discrepancies among studies, which may be due to differences in study design (Table 1). Therefore, we propose standardized experimental approaches to help identify generalities and contingencies in pathogen-vector-host interactions using avian malaria as a model (Figure 1).

\section{CURRENT EXPERIMENTAL APPROACHES}

In general, laboratory experiments assess mosquito attraction using dual-choice olfactometers, or they assess feeding choice by placing mosquitoes inside cages containing target host groups. Olfactometers expose mosquitoes to olfactory host cues $\left(\mathrm{CO}_{2}\right.$ and volatile compounds) and prevent mosquitoes from using visual cues or differences in body temperature for host detection (Lalubin et al., 2012; De Moraes et al., 2014; Díez-Fernández et al., 2020a). This approach is useful for studies using chemical stimuli collected from hosts and has the advantage to protect hosts from being bitten by mosquitoes. Host attractiveness in singlechoice system can be measured as the number of mosquitoes approaching a host in one end of an enclosure or in a tunnel in relation to the number of mosquitoes released at the opposite end (Cator et al., 2013; Batista et al., 2014).

Feeding rates can be measured by comparing the proportion of mosquitoes taking a blood meal from single-housed (Cornet et al., 2019) or pair-housed hosts. In the latter, two hosts from different groups (e.g., control vs. experimental group) are placed inside the same cage where mosquitoes are released and host choice is assessed via microsatellite testing (Yan et al., 2018b) or by molecular sexing methods (Cornet et al., 2013a; Yan et al., 2018a) using engorged mosquitoes to determine blood source. Overall, studies varied in duration and type (attraction assays using olfactometers vs. feeding choice inside cages), with feeding experiments used either immobilized or non-immobilized birds (Table 1). These differences, therefore, may explain discrepancies in the general findings.

\section{INDIRECT AND DIRECT MANIPULATION OF VECTOR'S BEHAVIOR BY PATHOGENS}

Pathogens can modify conditions within vertebrate hosts or within vectors to increase transmission rates from infected to uninfected hosts. These indirect and direct vector manipulations, respectively, may affect host-seeking behaviors, feeding behaviors, or both (Gandon, 2018).

\section{Infected Hosts and Mosquito Attraction}

Most studies tested whether hosts infected with Plasmodium parasites are more attractive to uninfected mosquitoes or whether uninfected mosquitoes feed more on infected hosts (indirect vector manipulation). Some of these studies also tested whether hosts with higher number of circulating gametocytes (sexual parasite stage that is infective to mosquitoes) were more attractive to mosquitoes even when compared with hosts with low levels of gametocytes (Table 1).

\section{Avian Malaria}

Cornet et al. (2013a,b) found that laboratory-reared Cx. pipiens fed more on domestic canaries (Serinus canaria) infected with a laboratory strain of Plasmodium relictum (SGS1) when compared to uninfected birds. This effect was detected in birds undergoing chronic infections (24-26 days post infection - dpi), but not in birds undergoing the acute phase of the infection (10 dpi; Cornet et al., 2013b). Mosquitoes fed more on birds with a higher hematocrit, a measure that can be interpreted as a higher blood quality (Cornet et al., 2013a).

Four avian malaria studies used wild caught $C x$. pipiens and free-living birds infected with Plasmodium spp. and uninfected birds to assess vector manipulation in more natural systems (Table 1). One set of experiments conducted by Yan et al. (2018b) compared infected birds with their counterparts treated with primaquine to reduce parasitemia (the number of circulating parasites). They found mosquitoes fed more on infected birds than on treated birds, which provided experimental support for the vector manipulation hypothesis. Similarly, mosquitoes were more attracted to body odors isolated from infected birds when compared to uninfected birds (Díez-Fernández et al., 2020b). However, this effect was not observed when using uropygial gland secretion collected from the same bird groups. Other experiments found that mosquitoes were either more attracted to uninfected birds (Lalubin et al., 2012) or that infection status did not change mosquito feeding rates when compared to control groups (Yan et al., 2018b; Gutiérrez-López et al., 2019a).

From the discussed studies, two used dual-choice olfactometers to assess mosquito attraction to birds or to chemical compounds collected from birds, while three studies released mosquitoes inside cages containing single birds or pairs of infected and uninfected birds that were immobilized in most cases (except Yan et al., 2018b; Table 1). Observed contrasting results addressing the host manipulation hypothesis may be the result of differences in host, vector and parasites used and/or due to differences in experimental settings. 
TABLE 1 | Studies conducted on mosquito attraction toward bird, humans and mice.

Plasmodium Infections

\begin{tabular}{|c|c|c|c|c|c|c|}
\hline \multicolumn{7}{|c|}{ Indirect manipulation } \\
\hline Host sp. & Vector sp.* & Parasite species & Factor & Main Results & Experimental details & Source ${ }^{\star \star}$ \\
\hline \multicolumn{7}{|c|}{ Avian hosts } \\
\hline $\begin{array}{l}\text { Great tit - Parus } \\
\text { major }\end{array}$ & $\begin{array}{l}\text { Culex pipiens (wild caught). Not } \\
\text { tested for pipiens and molestus } \\
\text { forms and hybrids. }\end{array}$ & $\begin{array}{l}\text { Plasmodium sp. - } \\
\text { natural infections }\end{array}$ & $\begin{array}{l}\text { Mosquito attraction } \\
\text { as a function of bird } \\
\text { infection. }\end{array}$ & $\begin{array}{l}\text { Infected birds less attractive than } \\
\text { uninfected birds. Females more } \\
\text { attractive to mosquitoes than males. }\end{array}$ & $\begin{array}{l}\text { Dual-choice olfactometer. Twenty-eight paired } \\
\text { experiments combining six infected and five } \\
\text { uninfected birds. Birds kept out of sight of the } \\
\text { mosquitoes. Parasitemia not assessed. } \\
\text { Experiments lasted } 15 \text { min. }\end{array}$ & $\begin{array}{l}\text { Lalubin et al. } \\
(2012)^{1}\end{array}$ \\
\hline $\begin{array}{l}\text { Domestic canary - } \\
\text { Serinus canaria }\end{array}$ & Cx. pipiens (S-LAB strain) & $\begin{array}{l}\text { Plasmodium } \\
\text { relictum (SGS1) - } \\
\text { experimental } \\
\text { infection }\end{array}$ & $\begin{array}{l}\text { Feeding choice as } \\
\text { a function of bird } \\
\text { infection and } \\
\text { infection stage } \\
\text { (acute or chronic). }\end{array}$ & $\begin{array}{l}\text { Mosquitoes fed more on chronically } \\
\text { infected birds than on either uninfected } \\
\text { or acutely infected birds. Mosquito } \\
\text { preference for birds with higher } \\
\text { hematocrit. No difference between } \\
\text { uninfected birds and birds during acute } \\
\text { infection. }\end{array}$ & $\begin{array}{l}\text { Paired birds in } 25 \text { independent cages with } 70 \\
\text { mosquitoes each. Feeding experiments lasted } \\
2 \mathrm{~h} \text {. Birds were immobilized. }\end{array}$ & $\begin{array}{l}\text { Cornet et al. } \\
(2013 b)^{2}\end{array}$ \\
\hline $\begin{array}{l}\text { House sparrow - } \\
\text { Passer domesticus }\end{array}$ & $\begin{array}{l}\text { Cx. pipiens - wild caught. Not } \\
\text { tested for pipiens and molestus } \\
\text { forms and hybrids. }\end{array}$ & $\begin{array}{l}\text { Plasmodium sp. - } \\
\text { natural infections }\end{array}$ & $\begin{array}{l}\text { Feeding choice as } \\
\text { a function of bird } \\
\text { infection. }\end{array}$ & $\begin{array}{l}\text { No difference in feeding rates between } \\
\text { infected and uninfected birds. }\end{array}$ & $\begin{array}{l}\text { Twenty pairs of infected and uninfected birds } \\
\text { exposed to mosquitoes. Birds were not } \\
\text { immobilized. Experiments lasted } 12 \mathrm{~h} \text {. }\end{array}$ & $\begin{array}{l}\text { Yan et al. } \\
(2018 b)^{3}\end{array}$ \\
\hline $\begin{array}{l}\text { House sparrow - } \\
\text { Passer domesticus }\end{array}$ & $\begin{array}{l}\text { CX. pipiens - wild caught. Not } \\
\text { tested for pipiens and molestus } \\
\text { forms and hybrids. }\end{array}$ & $\begin{array}{l}\text { Plasmodium sp. - } \\
\text { natural infections }\end{array}$ & $\begin{array}{l}\text { Feeding choice as } \\
\text { a function of } \\
\text { parasite levels. }\end{array}$ & $\begin{array}{l}\text { Higher feeding rates on non-treated } \\
\text { (higher parasite levels) than on treated } \\
\text { birds. Higher feeding rates on females. }\end{array}$ & $\begin{array}{l}\text { Parasitemia reduced via primaquine treatment. } \\
\text { Nineteen pairs of infected non-treated birds and } \\
\text { infected treated birds exposed to an average of } \\
151 \text { mosquitoes. Birds were not immobilized. } \\
\text { Experiments lasted } 12 \mathrm{~h} \text {. }\end{array}$ & $\begin{array}{l}\text { Yan et al. } \\
(2018 b)^{4}\end{array}$ \\
\hline $\begin{array}{l}\text { Jackdaw - Corvus } \\
\text { monedula, and } \\
\text { house sparrow - } \\
\text { Passer domesticus }\end{array}$ & $\begin{array}{l}\text { Wild caught Ochlerotatus } \\
\text { (Aedes) caspius and Cx. pipiens } \\
\text { (not tested for pipiens and } \\
\text { molestus forms and hybrids). }\end{array}$ & $\begin{array}{l}\text { Plasmodium sp. - } \\
\text { natural infections }\end{array}$ & $\begin{array}{l}\text { Feeding choice as } \\
\text { a function of bird } \\
\text { infection, sex and } \\
\text { body mass }\end{array}$ & $\begin{array}{l}\text { No difference in feeding rates for both } \\
\text { mosquito species according to } \\
\text { Plasmodium infection status and body } \\
\text { mass for both bird species. } \\
\text { Ochlerotatus caspius fed more on } \\
\text { female than on male jackdaws. }\end{array}$ & $\begin{array}{l}\text { Birds single-housed. Parasitemia not assessed. } \\
\text { Experiments lasted } 30 \text { min. Birds were } \\
\text { immobilized. }\end{array}$ & $\begin{array}{l}\text { Gutiérrez- } \\
\text { López et al. } \\
(2019 a)^{5}\end{array}$ \\
\hline
\end{tabular}


TABLE 1 | Continued

Plasmodium Infection

Indirect manipulation

Host sp.

Vector sp.*

Parasite species

Main Results

Experimental details

Mice - Mus

musculus

Anopheles stephensi

Plasmodium

chabaudi - clones

$\mathrm{CR}$ and $\mathrm{ER}$

function of host infection.

Plasmodium chabaudi - clone

Mice-Mus

Anopheles stephens

Humans - Homo sapiens (children)

Anopheles gambiae - ICIPE lab strain

Plasmodium

falciparum - natural infections

Plasmodium vivax natural infections
Mosquito attraction Gametocyte-positive mice after acute as a function of host infection and infection stage.

Mosquito attraction as a function of infection an gametocyte presence.

Mosquito attraction as a function of gametocyte resence in infected humans. tage more attractive

vs. chronically infected

gametocyte-negative mice). volatile profiles due to infection.

Children harboring gametocytes more and to infected children harboring asexual parasite stages only. No

difference in attractiveness after antimalarial treatment.

Patients harboring gametocytes more attractive when compared to patients with no visible gametocytes and when compared to patients during and after antimalarial treatment.

\section{Higher rates of mosquito feeding on}

sapiens (adults)

Anopheles darlingi infected hosts when compared to

uninfected hosts. Positive relationship between parasitemia and feeding rates.

Attractiveness associated to changes in attractive when compared to uninfected

Groups of six mice uninfected, infected with either CR or ER or infected with a 1:1 mix of both Plasmodium clones. Mosquito exposure at $14 \mathrm{dpi}$. Asexual stages and gametocyte ratios calculated by microscopy. Anesthetized single-house mice exposed to 20-40 mosquitoes. Experiments lasted $20 \mathrm{~min}$

Dual-choice assay in wind tunnel. Six pairs of infected and uninfected mice exposed to 20 mosquitoes for $15 \mathrm{~min}$.

Children divided into three categories: uninfected, infected presenting asexual parasite (2005)

Lacroix et al. forms only, and infected presenting gametocytes. One hundred mosquitoes released at the central chamber of three-way olfactometers in 12 experiments containing one child of each category. Experiments lasted $30 \mathrm{~min}$.

One foot of $P$. vivax-infected patients exposed to $10 F_{1}$ mosquitoes in single-choice

olfactometers (three replicates each time) for

$10 \mathrm{~min}$. A subset of these patients were exposed to mosquitoes during and after antimalarial therapy ( 7 and 14 days after

diagnosis, respectively). Gametocyte presence evaluated by microscopy only. 
Plasmodium Infections

\section{Indirect manipulation}

\begin{tabular}{|c|c|c|c|c|c|c|}
\hline Host sp. & Vector sp.* & Parasite species & Factor & Main Results & Experimental details & Source ${ }^{\star \star}$ \\
\hline \multicolumn{7}{|c|}{ Mammal hosts } \\
\hline $\begin{array}{l}\text { Humans - Homo } \\
\text { sapiens (children) }\end{array}$ & $\begin{array}{l}\text { Anopheles gambiae - Mbita } \\
\text { strain }\end{array}$ & $\begin{array}{l}\text { Plasmodium } \\
\text { falciparum - natural } \\
\text { infections }\end{array}$ & $\begin{array}{l}\text { Mosquito attraction } \\
\text { as a function of } \\
\text { gametocyte } \\
\text { presence and } \\
\text { intensity in infected } \\
\text { humans. }\end{array}$ & $\begin{array}{l}\text { Children with gametocytes detected by } \\
\text { microscopy (higher intensity) more } \\
\text { attractive than the other three groups } \\
\text { (parasite free, asexual stages only and } \\
\text { submicroscopic gametocyte levels). } \\
\text { Similar attractiveness among all groups } \\
\text { after treatment. }\end{array}$ & $\begin{array}{l}\text { Children divided into four groups: Uninfected, } \\
\text { asexual parasite stage only, gametocyte detected } \\
\text { by qPCR only, and gametocyte detected by } \\
\text { microscopy and by qPCR. One hundred } \\
\text { mosquitoes in a dual-choice system. Experiments } \\
\text { lasted } 30 \text { min and were repeated } 3 \text { weeks after } \\
\text { antimalarial treatment. }\end{array}$ & $\begin{array}{l}\text { Busula et al. } \\
\text { (2017) }\end{array}$ \\
\hline $\begin{array}{l}\text { Humans - Homo } \\
\text { sapiens (adults) }\end{array}$ & Anopheles coluzzii - lab colony & $\begin{array}{l}\text { Plasmodium } \\
\text { falciparum NF54 } \\
\text { strain - } \\
\text { experimental } \\
\text { infections }\end{array}$ & $\begin{array}{l}\text { Mosquito attraction } \\
\text { as a function of } \\
\text { host infection. }\end{array}$ & $\begin{array}{l}\text { Odor samples from infected individuals } \\
\text { less attractive when compared to } \\
\text { treated and to uninfected individuals in } \\
\text { one trial. No difference observed in the } \\
\text { second trial. }\end{array}$ & $\begin{array}{l}\text { Groups of } 30 \text { mosquitoes exposed to cotton pads } \\
\text { with odor from infected humans and with ammonia } \\
\text { (negative control) in a dual-choice system for } \\
15 \text { min. Odor samples from infected humans } \\
\text { collected before, during ( } 6-8 \text { dpi) and after ( } 34 \text { dpi) } \\
\text { treatment with antimalarial. }\end{array}$ & $\begin{array}{l}\text { de Boer et al. } \\
\text { (2017) }\end{array}$ \\
\hline $\begin{array}{l}\text { Humans - Homo } \\
\text { sapiens (children - } \\
\text { body odor) }\end{array}$ & $\begin{array}{l}\text { Anopheles gambiae - Mbita } \\
\text { strain }\end{array}$ & $\begin{array}{l}\text { Plasmodium } \\
\text { falciparum - natural } \\
\text { infection }\end{array}$ & $\begin{array}{l}\text { Mosquito } \\
\text { attractiveness of } \\
\text { foot odor as a } \\
\text { function of host } \\
\text { infection. }\end{array}$ & $\begin{array}{l}\text { Odors from infected children more } \\
\text { attractive when compared to odor from } \\
\text { the same children after parasite } \\
\text { clearance with antimalarials. No effect } \\
\text { of gametocyte presence or absence. }\end{array}$ & $\begin{array}{l}\text { Groups of } 10 \text { mosquitoes placed in a dual-choice } \\
\text { system containing socks worn by the same child } \\
\text { before and after antimalarial treatment (six } \\
\text { replicates). A total } 23 \text { children presented circulating } \\
\text { gametocytes (qPCR testing), } 10 \text { did not present } \\
\text { circulating gametocytes (microscopy testing) and } \\
12 \text { were not infected (qPCR testing). Experiments } \\
\text { lasted } 15 \text { min. }\end{array}$ & $\begin{array}{l}\text { Robinson et al. } \\
\text { (2018) }\end{array}$ \\
\hline
\end{tabular}

\section{Direct manipulation}

\begin{tabular}{|c|c|c|c|c|c|c|}
\hline Host sp. & Vector sp.* & Parasite species & Factor & Main Results & Experimental details & Source ${ }^{\star *}$ \\
\hline \multicolumn{7}{|c|}{ Avian hosts } \\
\hline $\begin{array}{l}\text { Domestic canary - } \\
\text { Serinus canaria }\end{array}$ & CX. pipiens (S-LAB strain) & $\begin{array}{l}\text { Plasmodium } \\
\text { relictum (SGS1) - } \\
\text { experimental } \\
\text { infection }\end{array}$ & $\begin{array}{l}\text { Feeding rate of } \\
\text { sporozoite-infected } \\
\text { and uninfected } \\
\text { mosquitoes as } \\
\text { function of bird } \\
\text { infection }\end{array}$ & $\begin{array}{l}\text { Both infective and uninfected } \\
\text { mosquitoes feed more on chronically } \\
\text { infected birds when compared with } \\
\text { uninfected birds. Positive correlation } \\
\text { between hematocrit level and feeding } \\
\text { rate in infected birds for both infected } \\
\text { and uninfected mosquitoes }\end{array}$ & $\begin{array}{l}\text { Birds from different groups exposed to mosquitoes } \\
\text { in different cages. Ten cages with uninfected birds } \\
\text { and } 10 \text { cages with infected birds exposed to } 40 \\
\text { infected and } 40 \text { uninfected mosquitoes each. } \\
\text { Behavioral assay at peak of sporozoite presence in } \\
\text { mosquitoes, } 12-14 \text { dpbm. Behavioral assay } 53-55 \\
\text { dpi of bird host. Immobilized birds. Feeding } \\
\text { experiment lasted } 2 \mathrm{~h} \text {. }\end{array}$ & $\begin{array}{l}\text { Cornet et al. } \\
(2013 a)^{6}\end{array}$ \\
\hline $\begin{array}{l}\text { Domestic canary - } \\
\text { Serinus canaria }\end{array}$ & CX. pipiens (S-LAB strain) & $\begin{array}{l}\text { Plasmodium } \\
\text { relictum (SGS1) - } \\
\text { experimental } \\
\text { infection }\end{array}$ & $\begin{array}{l}\text { Feeding rate as a } \\
\text { function of } \\
\text { mosquito infectivity } \\
\text { (harboring } \\
\text { sporozoites). }\end{array}$ & $\begin{array}{l}\text { No difference in feeding rate between } \\
\text { infective and uninfected mosquitoes at } \\
\text { the end of the experiments ( } 3 \mathrm{~h} \text { ). } \\
\text { Uninfected mosquitoes feed faster than } \\
\text { infective mosquitoes. }\end{array}$ & $\begin{array}{l}\text { Six cages, each with an uninfected bird exposed to } \\
45 \text { uninfected and } 45 \text { sporozoite-infected } \\
\text { mosquitoes ( } 13 \mathrm{dpbm}) \text {. Immobilized birds. }\end{array}$ & $\begin{array}{l}\text { Cornet et al. } \\
(2019)^{7}\end{array}$ \\
\hline
\end{tabular}


TABLE 1 | Continued

Plasmodium Infection

Direct manipulation

\begin{tabular}{|c|c|c|c|c|c|c|}
\hline Host sp. & Vector sp.* & Parasite species & Factor & Main Results & Experimental details & Source ${ }^{\star *}$ \\
\hline \multicolumn{7}{|c|}{ Mammal hosts } \\
\hline $\begin{array}{l}\text { Humans - Homo } \\
\text { sapiens (adults) }\end{array}$ & $\begin{array}{l}\text { Anopheles stephensi (NIH } \\
\text { strain) }\end{array}$ & $\begin{array}{l}\text { Plasmodium yoelii } \\
\text { (clone } 17 \mathrm{XNL} \text { ) }\end{array}$ & $\begin{array}{l}\text { Host choice as a } \\
\text { function of } \\
\text { mosquito infection, } \\
\text { parasite stage and } \\
\text { immune challenge. }\end{array}$ & $\begin{array}{l}\text { Mosquitoes feeding on infected blood } \\
\text { less likely to fly toward a human during } \\
\text { oocyst stage and more likely to fly } \\
\text { toward a human during sporozoites } \\
\text { stage when compared to negative } \\
\text { controls. Same changes were observed } \\
\text { in mosquitoes infected with heat-killed } \\
\text { E. coli. }\end{array}$ & $\begin{array}{l}\text { Host-seeking was tested at short and long } \\
\text { ranges. Behavioral challenges in mosquitoes } \\
\text { injected with heat-killed E. coli. Trials conducted } \\
\text { over a } 1 \mathrm{~h} \text { period. }\end{array}$ & $\begin{array}{l}\text { Cator et al. } \\
\text { (2013) }\end{array}$ \\
\hline $\begin{array}{l}\text { Human - Homo } \\
\text { sapiens (odor) }\end{array}$ & $\begin{array}{l}\text { Anopheles gambiae - Ngousso } \\
\text { strain }\end{array}$ & $\begin{array}{l}\text { P. falciparum - } \\
\text { NF54 strain }\end{array}$ & $\begin{array}{l}\text { Landing behavior in } \\
\text { matrixes with } \\
\text { human odor as a } \\
\text { function of } \\
\text { sporozoite infection } \\
\text { in mosquitoes. }\end{array}$ & $\begin{array}{l}\text { Infective mosquitoes more attracted to } \\
\text { human odor when compared to } \\
\text { uninfected mosquitoes. }\end{array}$ & $\begin{array}{l}\text { Twenty mosquitoes of each group were } \\
\text { provided with a choice of matrixes with and } \\
\text { without human odor. Average landing rate } \\
\text { calculated for each matrix group over } 3 \text { min. }\end{array}$ & $\begin{array}{l}\text { Smallegange } \\
\text { et al. (2013) }\end{array}$ \\
\hline $\begin{array}{l}\text { Humans - Homo } \\
\text { sapiens }\end{array}$ & $\begin{array}{l}\text { Anopheles gambiae and An. } \\
\text { coluzzii - lab colonies } \\
\text { replenished with wild-caught } \\
\text { mosquitoes. An. arabiensis - } \\
\text { wild-caught. }\end{array}$ & $\begin{array}{l}\text { Plasmodium } \\
\text { falciparum - natural } \\
\text { infections. }\end{array}$ & $\begin{array}{l}\text { Host choice as a } \\
\text { factor of mosquito } \\
\text { infection and } \\
\text { parasite } \\
\text { developmental } \\
\text { stages } \\
\text { (non-infectious vs. } \\
\text { infectious). }\end{array}$ & $\begin{array}{l}\text { No difference in host choice between } \\
\text { infected and uninfected mosquitoes } \\
\text { and between mosquitoes harboring } \\
\text { either oocysts or sporozoites. }\end{array}$ & $\begin{array}{l}\text { Mosquitoes fed on blood containing active and } \\
\text { inactivated gametocytes from the same human } \\
\text { host. Mosquito host-seeking tested in } \\
\text { dual-choice system using humans, calf and } \\
\text { outdoor air (control) lasting } 30 \text { min. Twenty } \\
\text { infected and } 20 \text { uninfected mosquitoes tested } \\
\text { simultaneously. Eight experimental replicates } \\
\text { using } 9 \text { different gametocyte carriers. }\end{array}$ & $\begin{array}{l}\text { Nguyen et al. } \\
\text { (2017) }\end{array}$ \\
\hline
\end{tabular}

infectious). using 9 different gametocyte carriers.

\begin{tabular}{|c|c|c|c|c|c|c|}
\hline \multicolumn{7}{|c|}{ Ardooviruses intections } \\
\hline Host sp. & Vector sp. & Virus & Factor & Main Results & Experimental details & Source \\
\hline $\begin{array}{l}\text { House sparrow - } \\
\text { Passer domesticus }\end{array}$ & $\begin{array}{l}\text { Culex quinquefasciatus and } \\
\text { Culex tarsalis (lab colony) }\end{array}$ & $\begin{array}{l}\text { St. Louis } \\
\text { encephalitis virus } \\
\text { and Western } \\
\text { equine encephalitis } \\
\text { virus - laboratory } \\
\text { strains }\end{array}$ & $\begin{array}{l}\text { Mosquito attraction } \\
\text { as a function of } \\
\text { Arbovirus infection } \\
\text { in birds }\end{array}$ & $\begin{array}{l}\text { No difference in C. quinquefasciatus } \\
\text { attraction toward SLEV-infected birds. } \\
\text { No difference in C. tarsalis attraction } \\
\text { toward WEEV-infected birds. Culex } \\
\text { quinquefasciatus more attracted to } \\
\text { adults than to nestlings. }\end{array}$ & $\begin{array}{l}\text { Host attractiveness measured with } 10 \\
\text { mosquitoes in each trial in a } 50 \mathrm{~cm} \\
\text { single-choice vertical olfactometer (birds placed } \\
\text { at the bottom) for } 10 \mathrm{~min} \text {. }\end{array}$ & $\begin{array}{l}\text { Scott et al. } \\
(1990)^{8}\end{array}$ \\
\hline $\begin{array}{l}\text { Human - Homo } \\
\text { sapiens and } \\
\text { chicken - Gallus } \\
\text { gallus domesticus } \\
\text { (odors) }\end{array}$ & $\begin{array}{l}\text { Cx. pipiens form pipiens (wild } \\
\text { caught). }\end{array}$ & $\begin{array}{l}\text { West Nile virus } \\
\text { lineage } 2 \\
\text { (originated from } \\
\text { Greece) }\end{array}$ & $\begin{array}{l}\text { Host-seeking } \\
\text { response and host } \\
\text { choice as a } \\
\text { function of } \\
\text { mosquito infection }\end{array}$ & $\begin{array}{l}\text { WNV-infected mosquitoes had lower } \\
\text { host-seeking response when compared } \\
\text { to controls. No changes in flight } \\
\text { duration and blood-feeding propensity. }\end{array}$ & $\begin{array}{l}\text { Host-seeking response measured in an } \\
\text { olfactometer. Between } 53 \text { and } 57 \text { mosquitoes } \\
\text { from each group exposed to human and } \\
\text { chicken odors for } 7 \text { min. }\end{array}$ & $\begin{array}{l}\text { Vogels et al. } \\
(2017)\end{array}$ \\
\hline
\end{tabular}


TABLE 1 | Continued

\begin{tabular}{|c|c|c|c|c|c|}
\hline \multicolumn{6}{|c|}{ Uropygial secretion, volatile compounds and other attributes } \\
\hline Host sp. & Vector sp. & Factor & Main Results & Experimental details & Source \\
\hline $\begin{array}{l}\text { Crow - Corvus } \\
\text { brachyrhynchus }\end{array}$ & $\begin{array}{l}\text { Cx. pipiens } / C x \text {. restuans and } \\
\text { Aedes vexans (experiment } \\
\text { performed in the wild) }\end{array}$ & $\begin{array}{l}\text { Mosquito attraction toward } \\
\mathrm{CO}_{2} \text { baited traps as a } \\
\text { function of trap height and } \\
\text { presence of uropygial gland } \\
\text { secretion. }\end{array}$ & $\begin{array}{l}\text { Cx. pipiens/Cx. restuans more } \\
\text { attracted to traps at } 5 \mathrm{~m} \text { high with } \\
\text { uropygial gland secretion and dry ice } \\
\text { (source of } \mathrm{CO}_{2} \text { ) when compared to } \\
\text { traps with dry ice only (control group). } \\
\text { No difference when traps were placed } \\
\text { at } 1.5 \mathrm{~m} \text { high and in the attraction of } \\
\text { Ae. vexans at both heights. }\end{array}$ & $\begin{array}{l}\text { Study performed in Ontario, Canada. Uropygial } \\
\text { secretion from frozen glands applied to cotton } \\
\text { swabs. Swabs with and without uropygial gland } \\
\text { secretion were attached to CDC traps baited } \\
\text { with dry ice. Traps run at heights of } 1.5 \text { and } 5 \mathrm{~m} \\
\text { in a total of } 280 \text { trap catches. }\end{array}$ & $\begin{array}{l}\text { Russell and } \\
\text { Hunter (2005) }\end{array}$ \\
\hline $\begin{array}{l}\text { American robin - } \\
\text { Turdus migratorius }\end{array}$ & $\begin{array}{l}\text { Several species observed } \\
\text { during filming, including } C X \text {. } \\
\text { pipiens, } C x \text {. restuans, and } \\
\text { Aedes albopictus as the most } \\
\text { common }\end{array}$ & $\begin{array}{l}\text { Mosquito landing rates on } \\
\text { either adults or nestlings of } \\
\text { American robins at nests. }\end{array}$ & $\begin{array}{l}\text { Mosquitoes land more frequently on } \\
\text { nesting adults than on nestlings, and } \\
\text { parental brooding activities reduces } \\
\text { mosquitos landing rates on nestlings. }\end{array}$ & $\begin{array}{l}\text { Natural experiment using direct observations } \\
\text { via filming. }\end{array}$ & $\begin{array}{l}\text { Griffing et al. } \\
(2007)\end{array}$ \\
\hline $\begin{array}{l}\text { Blue birds - Sialia } \\
\text { sialis }\end{array}$ & $\begin{array}{l}\text { Culex quinquefasciatus (wild } \\
\text { caught) }\end{array}$ & $\begin{array}{l}\text { Feeding choice as a } \\
\text { function of bird's age } \\
\text { (mother vs. nestlings). }\end{array}$ & $\begin{array}{l}\text { No feeding preference between mother } \\
\text { and nestlings. Feeding upon mothers } \\
\text { decreased as age of the nestlings } \\
\text { increased. }\end{array}$ & $\begin{array}{l}\text { Mosquitoes introduced into nest boxes } \\
\text { containing mother and nestlings. Around } 22 \\
\text { mosquitoes per nest box. Exposure run } \\
\text { overnight. }\end{array}$ & $\begin{array}{l}\text { Burkett- } \\
\text { Cadena et al. } \\
\text { (2010) }\end{array}$ \\
\hline $\begin{array}{l}\text { Zebra finch - } \\
\text { Taeniopygia guttata }\end{array}$ & $\begin{array}{l}\text { Culex quinquefasciatus - } \\
\text { laboratory colony }\end{array}$ & $\begin{array}{l}\text { Feeding choice as a } \\
\text { function of corticosterone } \\
\text { level in birds }\end{array}$ & $\begin{array}{l}\text { Higher feeding rates on birds with } \\
\text { artificially elevated levels of } \\
\text { corticosterone. }\end{array}$ & $\begin{array}{l}\text { Birds received one or two implants of } \\
\text { corticosterone and were assigned to CORT+ } \\
\text { and CORT++ groups, respectively. Negative } \\
\text { control and birds from both CORT groups } \\
\text { exposed simultaneously to } 50 \text { mosquitoes for } \\
13 \mathrm{~h} \text {. Birds were not immobilized. }\end{array}$ & $\begin{array}{l}\text { Gervasi et al. } \\
(2016)^{11}\end{array}$ \\
\hline $\begin{array}{l}\text { House sparrows - } \\
\text { Passer domesticus }\end{array}$ & Cx. pipiens (Laboratory colony) & $\begin{array}{l}\text { Mosquito attraction toward } \\
\text { chemicals from uropygial } \\
\text { gland secretions as a } \\
\text { function of bird sex and age }\end{array}$ & $\begin{array}{l}\text { Mosquitoes more attracted to uropygial } \\
\text { gland secretions from adults over } \\
\text { nestlings. No preference for adults over } \\
\text { fledglings. No difference in preference } \\
\text { for adults, nestlings or fledglings when } \\
\text { using semi-volatile components } \\
\text { isolated from secretions. }\end{array}$ & $\begin{array}{l}\text { Use of dual olfactometer. Thirty mosquitoes for } \\
\text { each trial, which lasted } 45 \text { min. }\end{array}$ & $\begin{array}{l}\text { Garvin et al. } \\
\text { (2018a) }\end{array}$ \\
\hline $\begin{array}{l}\text { House sparrow - } \\
\text { Passer domesticus } \\
\text { European starling - } \\
\text { Sturnus vulgaris } \\
\text { American robin - } \\
\text { Turdus migratorius }\end{array}$ & Cx. pipiens (laboratory colony) & $\begin{array}{l}\text { Mosquito attraction to } \\
\text { chemicals from uropygial } \\
\text { gland secretions as a } \\
\text { function of bird species. }\end{array}$ & $\begin{array}{l}\text { Chemical composition of robins' } \\
\text { uropygial gland secretions was different } \\
\text { to those of sparrows and starlings. No } \\
\text { differences on CX. pipiens preference } \\
\text { for secretions of robins over those of } \\
\text { sparrows. However, CX. pipiens more } \\
\text { attracted to live starlings and starlings } \\
\text { secretions than to either live robins or } \\
\text { their secretions. }\end{array}$ & $\begin{array}{l}\text { Adult House sparrows, European starlings and } \\
\text { American robins. Thirty mosquitoes for each } \\
\text { trials, which lasted } 2 \mathrm{~h} \text {. }\end{array}$ & $\begin{array}{l}\text { Garvin et al. } \\
\text { (2018b) }\end{array}$ \\
\hline
\end{tabular}


TABLE 1 | Continued

\begin{tabular}{|c|c|c|c|c|c|}
\hline \multicolumn{6}{|c|}{ Uropygial secretion, volatile compounds and other attributes } \\
\hline Host sp. & Vector sp. & Factor & Main Results & Experimental details & Source \\
\hline $\begin{array}{l}\text { House sparrow - } \\
\text { Passer domesticus }\end{array}$ & $\begin{array}{l}\text { Cx. pipiens - wild caught (not } \\
\text { distinguished for pipiens and } \\
\text { molestus forms). }\end{array}$ & $\begin{array}{l}\text { Feeding choice as a } \\
\text { function of bird metabolic } \\
\text { rate. }\end{array}$ & $\begin{array}{l}\text { Mosquito feeding rate similar between } \\
\text { control and treatment group. Negative } \\
\text { association between mosquito feeding } \\
\text { preference and bird resting metabolic } \\
\text { rate. Positive association between } \\
\text { mosquito feeding preference and bird } \\
\text { body mass. }\end{array}$ & $\begin{array}{l}\text { Use of 2,4-dinitrophenol to induce higher } \\
\text { metabolic rate. Thirty juvenile House sparrows, } \\
21 \text { males and nine females. Trials included a } \\
\text { pair of birds exposed to an average of } 190 \\
\text { mosquitoes for } 12 \mathrm{~h} \text {. Birds were not } \\
\text { immobilized. }\end{array}$ & $\begin{array}{l}\text { Yan et al. } \\
(2018 a)^{12}\end{array}$ \\
\hline $\begin{array}{l}\text { Great tit - Parus } \\
\text { major }\end{array}$ & $\begin{array}{l}\text { Cx. pipiens (laboratory colony } \\
\text { established with local } \\
\text { mosquitoes }\end{array}$ & $\begin{array}{l}\text { Feeding choice as a } \\
\text { function of host sex. }\end{array}$ & $\begin{array}{l}\text { No difference in host choice due to } \\
\text { nestling sex }\end{array}$ & $\begin{array}{l}\text { Eighteen independent cages with a pair of } \\
14 \text { days old siblings, one male and on female. } \\
\text { Twenty-five uninfected mosquitoes in each } \\
\text { cage allowed to feed for } 1 \mathrm{~h} \text {. Host sex } \\
\text { determined by PCR. }\end{array}$ & $\begin{array}{l}\text { Cozzarolo et al. } \\
\text { (2019) }\end{array}$ \\
\hline $\begin{array}{l}\text { House sparrow- } \\
\text { Passer domesticus } \\
\text { (juveniles) }\end{array}$ & $\begin{array}{l}\text { Wild caught Ochlerotatus } \\
\text { (Aedes) caspius and Cx. pipiens } \\
\text { (not tested for pipiens and } \\
\text { molestus forms and hybrids) }\end{array}$ & $\begin{array}{l}\text { Mosquito attraction toward } \\
\text { uropygial gland secretion. }\end{array}$ & $\begin{array}{l}\text { No difference in mosquito attraction } \\
\text { between uropygial gland secretion and } \\
\text { negative controls. }\end{array}$ & $\begin{array}{l}\text { Dual choice (Y-shaped) olfactometer. Negative } \\
\text { controls consisted on air flow with no stimuli. } \\
\text { Each trial run with } 20 \text { mosquitoes ( } 6-15 \text { days } \\
\text { old) for } 15 \text { min. CX. pipiens assayed in } 21 \text { trials } \\
\text { and O. caspius assayed in } 20 \text { trials. }\end{array}$ & $\begin{array}{l}\text { Díez-Fernández } \\
\text { et al. }\left(2020 \text { a) }{ }^{10}\right.\end{array}$ \\
\hline $\begin{array}{l}\text { House sparrow - } \\
\text { Passer domesticus } \\
\text { (juveniles) }\end{array}$ & $\begin{array}{l}\text { Cx. pipiens - wild caught. Not } \\
\text { tested for pipiens and molestus } \\
\text { forms and hybrids. }\end{array}$ & $\begin{array}{l}\text { Mosquito attraction toward } \\
\text { uropygial gland secretions } \\
\text { and whole-body odor as a } \\
\text { function of Plasmodium } \\
\text { infection. }\end{array}$ & $\begin{array}{l}\text { Mosquitoes more attracted to } \\
\text { whole-body odors of infected sparrows } \\
\text { regardless of sex. No effect when using } \\
\text { uropygial secretions. The volatile } \\
\text { lipophilic fraction of the uropygial } \\
\text { secretion was not different between } \\
\text { infected and uninfected sparrows. }\end{array}$ & $\begin{array}{l}\text { Dual choice (Y-shaped) olfactometer. } \\
\text { Experimental pairs were always of the same } \\
\text { sex. Each trial run with } 20 \text { mosquitoes } \\
\text { (6-15 days old) for } 15 \text { min in complete } \\
\text { darkness. Analyzed whether Plasmodium spp. } \\
\text { infections alter chemical composition of } \\
\text { uropygial secretions. }\end{array}$ & $\begin{array}{l}\text { Díez-Fernández } \\
\text { et al. }(2020 \mathrm{~b})^{9}\end{array}$ \\
\hline $\begin{array}{l}\text { Chicken (Gallus } \\
\text { gallus domesticus), } \\
\text { pigeon (Columba } \\
\text { livia), and magpie } \\
\text { (Pica pica) }\end{array}$ & $\begin{array}{l}\text { Culex quinquefasciatus (Thai } \\
\text { strain) and Cx. pipiens molestus } \\
\text { (laboratory colony) }\end{array}$ & $\begin{array}{l}\text { Mosquito attraction toward } \\
\text { whole-body odor in the } \\
\text { presence or absence of an } \\
\text { extra source of } \mathrm{CO}_{2} \text {. }\end{array}$ & $\begin{array}{l}\text { Cx. quinquefasciatus more attracted to } \\
\text { both chicken sexes and to female } \\
\text { pigeons. No difference toward male } \\
\text { pigeons and magpies. Addition of } \\
\mathrm{CO}_{2} \text { increased attraction toward } \\
\text { chickens and pigeons, but not toward } \\
\text { magpies. Culex p. molestus more } \\
\text { attracted only to chickens (both sexes). } \\
\text { No effect on the addition of } \mathrm{CO}_{2} \text {. }\end{array}$ & $\begin{array}{l}\text { Single-choice and dual choice olfactometers } \\
\text { used for } C X \text {. quinquefasciatus and for } C X \text {. } \\
\text { p. molestus, respectively. Bird sex determined } \\
\text { only for chickens and pigeons. Ten trials } \\
\text { performed with } 30 \text { mosquitoes lasting } 4 \text { min for } \\
C x \text {. quinquefasciatus and } 2 \text { min for } C x \text {. } \\
\text { p. molestus. }\end{array}$ & $\begin{array}{l}\text { Spanoudis } \\
\text { et al. (2020) }\end{array}$ \\
\hline
\end{tabular}

No effect on the addition of $\mathrm{CO}_{2}$.

Cx. pipiens for Diptera taxonomists is still under debate, and sometimes is used as a synonym of Cx. quinquefasciatus. Some authors even write is as Cx. pipiens quinquefasciatus.

** Superscript numbers, whenever present, are related to the experiments described in Figure 1.

From the perspective of the vector, here we consider an indirect manipulation when the parasite is infecting the vertebrate host (i.e., the parasitic infection changes some trait of the host that makes it more attractive to the vector), and a direct manipulation when the parasite is inside the vector. 


\section{A}

Feeding choice experiments

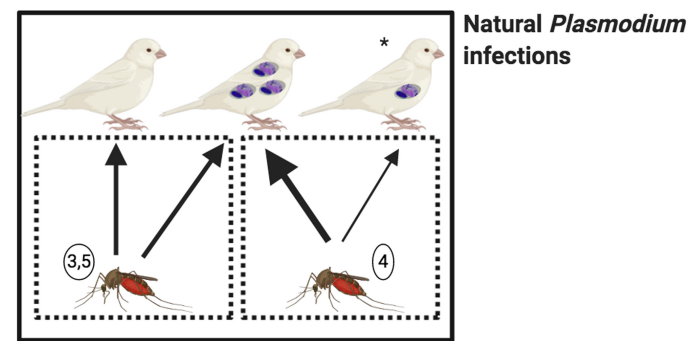

* Birds received antimalarial to reduce parasitemia

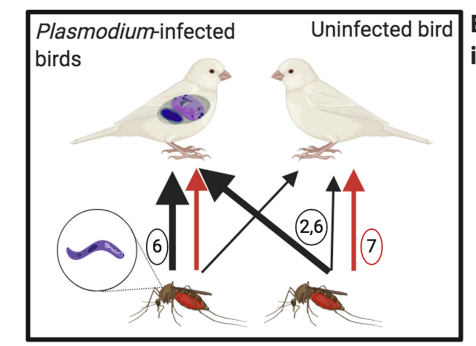
infections
Experimental Plasmodium c

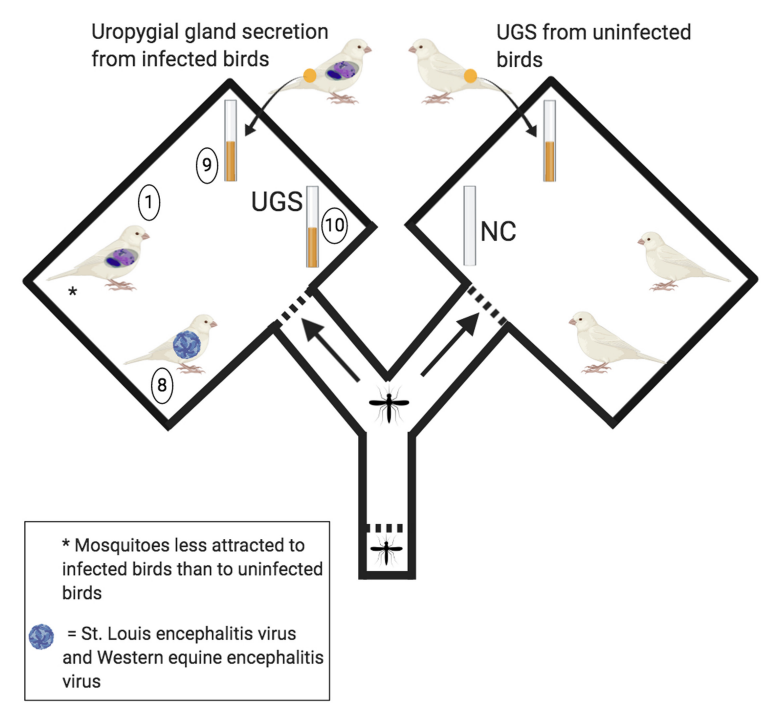

Feeding choice and host choice experiments with differences between target groups

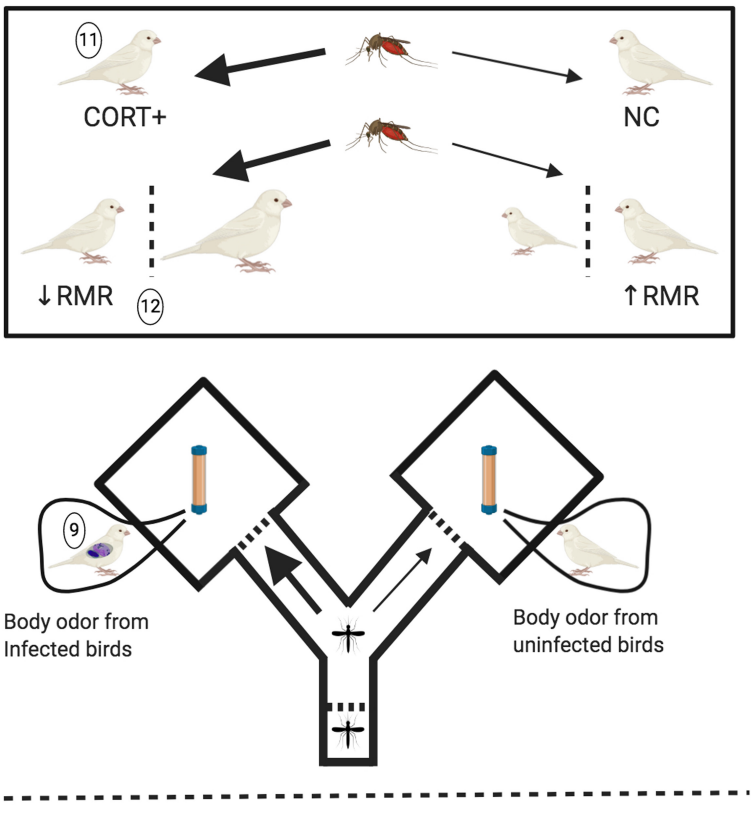

D

Directions for future studies

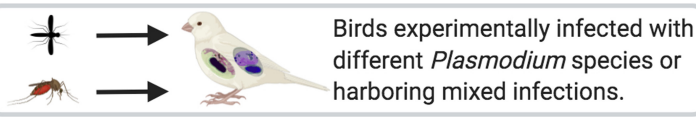

different Plasmodium species or

- Quantify levels of circulating gametocytes. Use the same bird during acute and chronic stages of infection.

atc

Infect wild-caught mosquitoes with Plasmodium from free-living birds. Mosquitoes at peak levels of sporozoite density.

Immobilized birds allow for shorter
times in feeding experiments. Run
attraction experiments for 15 min.

FIGURE 1 | Experimental designs to determine the factors potentially affecting mosquito feeding behavior and attraction toward birds. (A) Upper pane: mosquitoes do not feed more on naturally infected birds than on uninfected birds. However, feeding rates are higher on birds with higher levels of circulating parasites (thick black arrow) when compared to birds with reduced levels of parasites after antimalarial treatment (thin black arrow). Lower pane: uninfected mosquitoes feed more on birds experimentally infected with Plasmodium relictum (thick black arrow) when compared to uninfected birds. Infective mosquitoes (harboring sporozoites) may (thick black arrow) or may not (red arrows) feed more on infected birds than on uninfected ones. (B) Upper pane: Mosquitoes feed more (thick black arrows) on birds with higher levels of corticosterone (CORT+) and feed more on birds with lower resting metabolic rate (RMR) and with higher body mass. Lower pane: Mosquitoes are more attracted toward whole-body odor from birds naturally infected with Plasmodium parasites. (C) No mosquito preference in relation to bird arboviral infection and to uropygial gland secretion (UGS) from Plasmodium-infected birds when compared to UGS from uninfected birds. No mosquito preference toward UGS when compared to vials containing no stimuli. Mosquitos were less attracted to birds naturally infected with Plasmodium. (D) Open questions that can be addressed in future studies, always keeping in mind to standardize bird and mosquito cohorts in terms of age and sex. Numbers in circles refer to specific studies indicated in Table 1 (see Source column). Figure created with BioRender.com. 


\section{Human and Mice Malaria}

Most studies on human malaria found that natural Plasmodium infections are associated with higher host attractiveness to vectors. Three out of four studies found that Anopheles mosquitoes were more attracted to humans harboring microscopically detectable gametocytes when compared to uninfected people and to people with only asexual stages detectable in the blood stream (Lacroix et al., 2005; Batista et al., 2014; Busula et al., 2017). The fourth study showed that socks worn by children parasitized by Plasmodium falciparum were more attractive to mosquitoes when compared with socks worn by the same children after the completion of antimalarial treatment (Robinson et al., 2018). A study during its first trial using feet odors found that samples from individuals infected with $P$. falciparum were less attractive to mosquitoes when compared to cotton pads from the same individuals after antimalaria treatment and compared to odors from uninfected people; the second trial did not find any difference in mosquito attraction (de Boer et al., 2017).

Two studies using mice malaria found that Plasmodium chabaudi infection increased Anopheles stephensi feeding rates (Ferguson et al., 2003) and attraction (De Moraes et al., 2014) when compared to uninfected hosts. This latter study found this effect only during the initial chronic stage, which was associated to microscopically observable levels of circulating gametocytes. These studies on mammals also show contrasting results, precluding generalizations and strengthening the fact of having a standard initial experimental design.

\section{Infected Mosquitoes and Host-Feeding}

Pathogens can influence host-seeking and feeding behaviors during their development inside mosquitoes. Because mosquitoes only become infective a few days or even weeks after blood feeding on an Plasmodium-infected host (i.e., once sporozoites are found in salivary glands; Valkiūnas et al., 2013; Palinauskas et al., 2016), changes in host-seeking and feeding behaviors may be displayed differentially according to vector infective stage rather than infection status only.

When mosquitoes were simultaneously exposed to both Plasmodium-infected and uninfected birds, both infective and uninfected mosquitoes fed more frequently on Plasmodiuminfected birds (Cornet et al., 2013a). When exposed to uninfected birds, uninfected mosquitoes fed faster than infective mosquitoes, but the final feeding rate after the $3 \mathrm{~h}$ the experiment lasted was identical between both groups (Cornet et al., 2019). This demonstrates that experiment duration can affect results, because the authors would have observed higher feeding rates of uninfected mosquitoes if experiments had lasted only $1 \mathrm{~h}$.

Mosquitoes that fed on Plasmodium yoelii-infected mice were less attracted to hosts during the oocyst stage (non-infective) than during the sporozoite stage, when they engaged in higher rates of host-seeking behavior when compared to negative controls (Cator et al., 2013). However, these changes were also observed in mosquitoes that were not infected after feeding on infected mice. Therefore, the observed behavioral changes may be due to
Plasmodium infections and to unspecific immune stimuli in the challenged mosquitoes.

Laboratory reared Anopheles gambiae harboring sporozoites of a laboratory strain of $P$. falciparum were more attracted to human odor when compared to infective mosquitoes exposed to negative-controls (Smallegange et al., 2013). In contrast, three different species of wild caught Anopheles infected with $P$. falciparum from natural infections had similar attraction rates to humans when compared with uninfected mosquitoes (Nguyen et al., 2017).

\section{CHEMICAL COMPOUNDS STUDIED IN RELATION TO VECTOR ATTRACTION}

Differences in mosquito attraction are related to dissimilarities in chemical volatiles produced by each host, which can be modified by pathogen infections. Díez-Fernández et al. (2020b) were the first to show that whole-body odor from Plasmodiuminfected birds are more attractive to mosquitoes when compared to uninfected birds. De Moraes et al. (2014) demonstrated that mice displayed different odor profiles when infected with Plasmodium chabaudi and became more attractive to mosquitoes after the acute phase of infection. Mice produced fewer volatile chemicals during the acute phase of infection and attracted less mosquitoes. In humans, increased mosquito attraction toward Plasmodium-infected children was associated to changes in skin odor profile driven by increases in the production of aldehydes (Robinson et al., 2018). Children infected with $P$. falciparum in Malawi produced a different chemical breath composition than uninfected children (Schaber et al., 2018), which mosquitos can use as a cue to find their hosts (Kelly et al., 2015). Thus, the chemical volatiles emitted by hosts and how they change during infection likely constitute the most important factor determining vector attraction.

Birds' uropygial (or preen) gland produces some antimicrobial and antifungal chemicals that protect the plumage and skin of birds; they also provide defense against ectoparasites and haemosporidian infection (Salibian and Montalti, 2009; Magallanes et al., 2016). Some studies suggest that uropygial gland secretions can attract vectors (Russell and Hunter, 2005), but others suggest no increased attraction toward such secretions (Díez-Fernández et al., 2020a) or toward common chemicals from uropygial glands (Allan et al., 2006) when compared to negative controls (no stimuli). The amount of volatile compounds of uropygial glands differ among adult, fledging and nestling house sparrows (Passer domesticus), but these differences did not modify mosquito attraction in dual-choice olfactometer assays (Garvin et al., 2018a). Plasmodium infection did not change the composition of the volatile fraction of uropygial gland secretion in house sparrows when compared to the uninfected group, and this may explain the similar mosquito attraction toward the secretion from both bird groups (DíezFernández et al., 2020b). However, other study showed that exposure to Plasmodium altered the wax ester composition of the secretion in song sparrows (Melospiza melodia), regardless of whether infections were cleared or not (Grieves et al., 2018). 
Therefore, more research is needed to cover the whole array of chemicals produced by the uropygial gland and their effect on different vector species and families (Martínez-de la Puente et al., 2020). Particularly considering that parasites from different Haemosporida genera (e.g., Haemoproteus, Leucocytozoon) are transmitted by different Diptera families, which have both different life cycles and ecologies (Ibáñez-Bernal et al., 2020).

\section{STUDIES FOCUSED ON OTHER HOST ATTRIBUTES}

Physiological differences (e.g., hormones) and differential exposure between males and females due to sex-specific physiology (e.g., immunosuppression due to breeding activities), behavior (e.g., nesting), morphology (e.g., body size), and pathogen infections can influence mosquito attraction (Darbro et al., 2007; Cozzarolo et al., 2019). For instance, Cx. pipiens were more attracted to female Great tits (Parus major; Lalubin et al., 2012). Ochlerotatus caspius fed more on female than on male jackdaws (Corvus monedula), but no difference was observed when using $C x$. pipiens. In addition, these two mosquito species did not show sex-biased feeding preference toward house sparrows (Gutiérrez-López et al., 2019a). Cozzarolo et al. (2019) did not find any mosquito feeding preference between sexes, but they used nestlings instead of adults (see also Simpson et al., 2009). Higher feeding rate on female birds by wildcaught Culex restuans has been reported during the nesting period, suggesting the role of seasonality in this process (Egizi et al., 2014), but this has yet to be investigated under laboratory conditions. Yan et al. (2018a) found that mosquitos fed less on birds with higher resting metabolic rate, which authors interpreted as related to bird activity, where birds with higher metabolic rates are more active and thus display more defensive behaviors than more lethargic individuals. Finally, elevated levels of corticosterone increased bird attractiveness to mosquitoes despite the fact that these birds displayed increased anti-mosquito behaviors when compared to the control group (Gervasi et al., 2016). Increases in corticosterone levels may lead to Plasmodium resurgence in the blood stream in chronically infected birds with an increase in parasitemia (Applegate and Beaudoin, 1970). Therefore, the interaction between elevated stress hormone levels and parasitemia may synergistically increase bird attractiveness to mosquitoes, but this has yet to be tested. Different results across studies may be due to differences in life history traits of bird species, which may in turn influence exposure to vectors and species-specific physiological trade-offs. Hence, we suggest that fair comparisons and generalizations must be done across species (both hosts and vectors) with similar life history traits.

\section{SUMMARY OF CURRENT EVIDENCE AND IMPLICATIONS FOR FUTURE STUDIES}

We found that fewer studies assessed direct effects of avian and mammalian Plasmodium on mosquito host choice (infective mosquitoes) when compared to the number of studies on indirect effects on mosquitoes (infected vertebrate hosts). Rearing mosquitoes under laboratory conditions over many generations may alter some genetic traits involved in their responsiveness to host cues (Takken and Verhulst, 2013), but nothing is known about whether genetic bottlenecks would change as a function of host-vector-parasite lineage assemblages (Gutiérrez-López et al., 2020). Although using hosts (birds and mice), mosquitoes and pathogens maintained in laboratories has provided fundamental tests of hypothesis related to the vector manipulation by pathogens, experiments mimicking natural conditions using wild caught mosquitoes are essential to fill in gaps for real world situations.

Free-living birds are infected with genetic lineages that vary in their pathological effects as a function of host species and parasite lineage (Palinauskas et al., 2011; Himmel et al., 2020). Assays using experimental infections have focused on domestic canaries infected with a $P$. relictum strain (SGS1) kept in the laboratory over dozens of passages, while assays using free-living birds have used three avian species naturally infected with a high diversity of parasites. In Gutiérrez-López et al. (2019a), 21 of 23 jackdaws exposed to wild-caught $C x$. pipiens were naturally infected with $P$. relictum (SGS1), and yet these authors did not find differences in mosquito feeding rates between infected and uninfected birds. On the other hand, laboratory-reared mosquitoes fed more on canaries experimentally infected with a laboratory strain of $P$. relictum SGS1 (Cornet et al., 2013a,b).

The use of naturally infected birds has the limitation of confounding the bird's infection stage during mosquito exposure. This is because in birds the Plasmodium acute stage of infection usually finishes within a month post infection. After this period surviving birds enter the chronic stage of infection, in which they display low parasitemia for long periods or even for their entire life (Valkiūnas, 2005). In many of the studies, free-living birds were undergoing chronic infections when exposed to mosquitoes and parasitemia was not evaluated. Interestingly, mosquitoes were more attracted to chronically infected birds when compared to acute infection stages in experimental settings (Cornet et al., 2013b), which may be interpreted as a parasite avoidance strategy because high parasitemia in the host is associated to high vector mortality (Gutiérrez-López et al., 2019b).

Birds and mosquitoes can be co-infected by different genetically related or unrelated parasites (e.g., co-infection between Plasmodium parasites and WNV; Hughes et al., 2010; Medeiros et al., 2014). Co-infections by different Plasmodium spp. and other related haemosporidians are often found in wild birds (e.g., Valkiūnas et al., 2006). Yet, evidence for the effects of multiple infections on vector feeding behavior and on fitness of both bird hosts (e.g., Marzal et al., 2008) and vectors (e.g., Gutiérrez-López et al., 2020) is still very limited (see Martínez-de la Puente et al., 2020). To complicate matters further, if mosquitoes are more attracted to birds co-infected with Plasmodium and arboviruses than to birds infected with arboviruses only, avian malaria may indirectly increase the transmission of zoonotic pathogens. Future studies, therefore, should perform feeding or attraction assays using birds 
experimentally infected with strains of different Plasmodium species with as few laboratory passages as possible, and also using different parasite groups (e.g., malaria and arbovirus). In addition, stage of infection (acute vs. chronic) and gametocyte levels (parasitemia) should be taken into consideration in studies using both natural and experimental infections. Furthermore, mosquitoes captured from the wild should be favored over populations kept in the laboratory for many generations. This would provide more real settings to understand host attractiveness to free-moving mosquitoes in nature.

Important issues to consider when planning experiments are:

(1) will birds be free or immobilized? Free birds may reflect natural conditions, but immobilized birds will increase vector sample size and reduce vector mortality by birds' behavioral defenses. (2) Will birds be visible to vectors or just odor cues will be investigated? Our opinion is that light levels should reflect those from the natural conditions during which vector activity peaks, which depends on vector species. (3) What would be the duration of the experiment? Immobilizing birds allows for shorter feeding experiments $(1-3 \mathrm{~h})$, while 15 min seems to be a common and reasonable time for attraction experiments. Ideally, researchers must know the natural feeding ecology of the studied vector in order to make an informed decision on the duration of experiments. Finally, (4) co-infections by different Plasmodium lineages or different parasite genera (e.g., Plasmodium/Haemoproteus) that are ubiquitous in nature, or even by Haemosporidians and arboviruses (e.g., WNV and USUV) should be considered. Understanding factors that change mosquito attraction to birds may have implications for human health as well, since highly ornithophilic mosquitoes such as $C x$. pipiens, $C x$. perexiguus, and $C x$. restuans are vectors of some arboviruses

\section{REFERENCES}

Abella-Medrano, C. A., Ibáñez-Bernal, S., Carbó-Ramírez, P., and Santiago-Alarcon, D. (2018). Blood-meal preferences and avian malaria detection in mosquitoes (Diptera: Culicidae) captured at different land use types within a neotropical montane cloud forest matrix. Parasitol. Int. 67, 313-320. doi: 10.1016/j.parint.2018. 01.006

Allan, S. A., Bernier, U. R., and Kline, D. L. (2006). Laboratory evaluation of avian odors for mosquito (Diptera: Culicidae) attraction. J. Med. Entomol. 43, 225-231. doi: 10.1093/jmedent/43.2.225

Applegate, J. E., and Beaudoin, R. L. (1970). Mechanism of spring relapse in avian malaria: effect of gonadotropin and corticosterone. J. Wildl. Dis. 6, 443-447. doi: 10.7589/0090-3558-6.4.443

Atkinson, C. T., and Van Riper, I. I. I. C. (1991). "Pathogenicity and epizootiology of avian haematozoa: Plasmodium, Leucocytozoon and Haemoproteus," in BirdParasite Interacctions, eds J. E. Loye and M. Zuk (Oxford: Oxford University Press), 19-48.

Batista, E. P., Costa, E. F., and Silva, A. A. (2014). Anopheles darlingi (Diptera: Culicidae) displays increased attractiveness to infected individuals with Plasmodium vivax gametocytes. Parasit. Vector. 7:251. doi: 10.1186/1756-33057-251

Brugman, V. A., Hernández-Triana, L. M., Medlock, J. M., Fooks, A. R., Carpenter, S., and Johnson, N. (2018). The role of Culex pipiens L. (Diptera: Culicidae) in virus transmission in Europe. Int. J. Environ. Res. Public Health 15:389. doi: 10.3390/ijerph15020389 that may affect humans, such as WNV and USUV (e.g., Kilpatrick et al., 2006; Vázquez et al., 2011; Brugman et al., 2018). Thus, further studies are necessary to understand the range of effects that multiple infections may have on host-vector contact rates and, hence, on the dynamics of parasite transmission. Our review highlights the need to design and implement general standard experimental procedures to obtain comparable results, which can help to generalize findings across studies and to identify contingencies (Figure 1) that later will allow drawing generalizations by, for instance, conducting meta-analyses.

\section{AUTHOR CONTRIBUTIONS}

Both authors have made a substantial, direct and intellectual contribution to the work, and approved it for publication.

\section{FUNDING}

DS-A has been supported by Consejo Nacional de Ciencia y Tecnología (CONACYT, project number Ciencia Básica 201101-168524 and project number Problemas Nacionales 201501-1628). FCF was supported by National Science Foundation grant 1717498 as part of the joint NSF-NIH-USDA Ecology and Evolution of Infectious Diseases program.

\section{ACKNOWLEDGMENTS}

We thank Sergio Ibáñez-Bernal, Daniela A. Dutra, Dina M. Fonseca, the editor Laura Gangoso and the three reviewers for their comments that highly improved the quality of this review.

Burkett-Cadena, N. D., Ligon, R. A., Liu, M., Hassan, H. K., Hill, G. E., Eubanks, M. D., et al. (2010). Vector-host interactions in avian nests: do mosquitoes prefer nestlings over adults? Am. J. Trop. Med. Hyg. 83, 395-399. doi: 10.4269/ ajtmh.2010.10-0048

Busula, A. O., Bousema, T., Mweresa, C. K., Masiga, D., Logan, J. G., Sauerwein, R. W., et al. (2017). Gametocytemia and attractiveness of Plasmodium falciparum-infected kenyan children to Anopheles gambiae mosquitoes. J. Infect. Dis. 216, 291-295. doi: 10.1093/infdis/jix214

Cator, L. J., George, J., Blanford, S., Murdock, C. C., Baker, T. C., Read, A. F., et al. (2013). 'Manipulation' without the parasite: altered feeding behaviour of mosquitoes is not dependent on infection with malaria parasites. Proc. R. Soc. B 280:20130711. doi: 10.1098/rspb.2013.0711

Cornet, S., Nicot, A., Rivero, A., and Gandon, S. (2013a). Both infected and uninfected mosquitoes are attracted toward malaria infected birds. Malar. J. 12:179. doi: 10.1186/1475-2875-12-179

Cornet, S., Nicot, A., Rivero, A., and Gandon, S. (2013b). Malaria infection increases bird attractiveness to uninfected mosquitoes. Ecol. Lett. 16, 323-329. doi: $10.1111 /$ ele.12041

Cornet, S., Nicot, A., Rivero, A., and Gandon, S. (2019). Avian malaria alters the dynamics of blood feeding in Culex pipiens mosquitoes. Malar. J. 18, 82. doi: 10.1186/s12936-019-2690-5

Cozzarolo, C.-S., Sironi, N., Glaizot, O., Pigeault, R., and Christe, P. (2019). Sex-biased parasitism in vector-borne disease: vector preference? PLoS One 14:e0216360. doi: 10.1371/journal.pone.0216360

Darbro, J. M., Dhondt, A. A., Vermeylen, F. M., and Harrington, L. C. (2007). Mycoplasma gallisepticum infection in house finches (Carpodacus mexicanus) 
affects mosquito blood feeding patterns. Am. J. Trop. Med. Hyg. 77, 488-494. doi: 10.4269/ajtmh.2007.77.488

de Boer, J. G., Robinson, A., Powers, S. J., Burgers, S. L. G. E., Caulfield, J. C., Birkett, M. A., et al. (2017). Odours of Plasmodium falciparum-infected participants influence mosquito-host interactions. Sci. Rep. 7:9283. doi: 10.1038/s41598017-08978-9

De Moraes, C. M., Stanczyk, N. M., Betz, H. S., Pulido, H., Sim, D. G., Read, A. F., et al. (2014). Malaria-induced changes in host odors enhance mosquito attraction. Proc. Natl. Acad. Sci. U.S.A. 111, 11079-11084. doi: 10.1073/pnas. 1405617111

Díez-Fernández, A., Martínez-de la Puente, J., Gangoso, L., Ferraguti, M., Soriguer, R., and Figuerola, J. (2020a). House sparrow uropygial gland secretions do not attract ornithophilic nor mammophilic mosquitoes. Med. Vet. Entomol. 2020:12401. doi: 10.1111/mve.12401

Díez-Fernández, A., Martínez-de la Puente, J., Gangoso, L., López, P., Soriguer, R., Martín, J., et al. (2020b). Mosquitoes are attracted by the odour of Plasmodiuminfected birds. Int. J. Parasitol. 50, 569-575. doi: 10.1016/j.ijpara.2020.03. 013

Egizi, A. M., Farajollahi, A., and Fonseca, D. M. (2014). Diverse host feeding on nesting birds may limit early-season West Nile virus amplification. Vector Borne Zoonotic Dis. 14, 447-453. doi: 10.1089/vbz.2013.1536

Farajollahi, A., Fonseca, D. M., Kramer, L. D., and Marm Kilpatrick, A. (2011). "Bird biting" mosquitoes and human disease: a review of the role of Culex pipiens complex mosquitoes in epidemiology. Infect. Genet. Evol. 11, 1577-1585. doi: 10.1016/j.meegid.2011.08.013

Ferguson, H. M., Rivero, A., and Read, A. F. (2003). The influence of malaria parasite genetic diversity and anaemia on mosquito feeding and fecundity. Parasitology 127, 9-19. doi: 10.1017/s0031182003003287

Gandon, S. (2018). Evolution and manipulation of vector host choice. Am. Nat. 192, 23-34. doi: 10.1086/697575

Garvin, M. C., Austin, A., Boyer, K., Gefke, M., Wright, C., Pryor, Y., et al. (2018a). Attraction of Culex pipiens to house sparrows is influenced by host age but not uropygial gland secretions. Insects 9:127. doi: 10.3390/insects9040127

Garvin, M. C., Austin, A. L., Stracker, N. H., Slowinski, S. P., Rutter, J. E., Butler, M., et al. (2018b). Attraction of Culex pipiens to uropygial gland secretions does not explain feeding preference for American robins. J. Vect. Ecol. 43, 110-116. doi: $10.1111 /$ jvec. 12290

Gervasi, S. S., Burkett-Cadena, N., Burgan, S. C., Schrey, A. W., Hassan, H. K., Unnasch, T. R., et al. (2016). Host stress hormones alter vector feeding preferences, success, and productivity. Proc. R. Soc. B 283:20161278. doi: 10. 1098/rspb.2016.1278

Grieves, L. A., Kelly, T. R., Bernards, M. A., and MacDougall-Shackleton, E. A. (2018). Malarial infection alters wax ester composition of preen oil in songbirds: results of an experimental study. Auk: Ornithol. Adv. 135, 767-776. doi: 10. 1642/AUK-17-242.1

Griffing, S. M., Kilpatrick, A. M., Clark, L., and Marra, P. P. (2007). Mosquito landing rates on nesting American robins (Turdus migratorius). Vect. Borne Zoonot. Dis. 7, 437-443. doi: 10.1089/vbz.2006.0560

Gutiérrez-López, R., Martínez-de la Puente, J., Gangoso, L., Soriguer, R., and Figuerola, J. (2019a). Effects of host sex, body mass and infection by avian Plasmodium on the biting rate of two mosquito species with different feeding preferences. Parasit. Vect. 12:87. doi: 10.1186/s13071-019-3342-x

Gutiérrez-López, R., Martínez-de la Puente, J., Gangoso, L., Yan, J., Soriguer, R., and Figuerola, J. (2019b). Experimental reduction of host Plasmodium infection load affects mosquito survival. Sci. Rep. 9:8782. doi: 10.1038/s41598019-45143-w

Gutiérrez-López, R., Martínez-de la Puente, J., Gangoso, L., Soriguer, R., and Figuerola, J. (2020). Plasmodium transmission differs between mosquito species and parasite lineages. Parasitology 147, 441-447. doi: 10.1017/ S0031182020000062

Heil, M. (2016). Host manipulation by parasites: cases, patterns and remaining doubts. Front. Ecol. Evol. 4:80. doi: 10.3389/fevo.2016.00080

Himmel, T., Harl, J., Pfanner, S., Nedorost, N., Nowotny, N., and Weissenböck, H. (2020). Haemosporidioses in wild Eurasian blackbirds (Turdus merula) and song thrushes (T. philomelos): an in situ hybridization study with emphasis on exo-erythrocytic parasite burden. Malar. J. 19:69. doi: 10.1186/s12936-0203147-6
Hughes, T., Irwin, P., Hofmeister, E., and Paskewitz, S. M. (2010). Occurrence of avian Plasmodium and West Nile virus in Culex species in Wisconsin. J. Am. Mosq. Control Assoc. 26, 24-31. doi: 10.2987/09-5893.1

Ibáñez-Bernal, S., Rivera-García, K. D., and Abella-Medrano, C. A. (2020). "Introduction to the taxonomy and general biology of Diptera (Insecta) involved in the transmission of avian haemosporida," in Avian Malaria and Related Parasites in the Tropics - Ecology, Evolution and Systematics, eds D. Santiago-Alarcon and A. Marzal (Cham: Springer).

Kelly, M., Su, C.-Y., Schaber, C., Crowley, J. R., Hsu, F.-F., Carlson, J. R., et al. (2015). Malaria parasites produce volatile mosquito attractants. mBio 6:e0023515.

Kilpatrick, A. M., Kramer, L. D., Jones, M. J., Marra, P. P., and Daszak, P. (2006). West Nile virus epidemics in North America are driven by shifts in mosquito feeding behavior. PLoS Biol. 4:e82. doi: 10.1371/journal.pbio.0040082

Lacroix, R., Mukabana, W. R., Gouagna, L. C., and Koella, J. C. (2005). Malaria infection increases attractiveness of humans to mosquitoes. PLoS Biol. 3:e298. doi: 10.1371/journal.pbio.0030298

Lalubin, F., Bize, P., van Rooyen, J., Christe, P., and Glaizot, O. (2012). Potential evidence of parasite avoidance in an avian malarial vector. Anim. Behav. 84, 539-545. doi: 10.1016/j.anbehav.2012.06.004

Magallanes, S., Møller, A. P., García-Longoria, L., de Lope, F., and Marzal, A. (2016). Volume and antimicrobial activity of secretions of the uropygial gland are correlated with malaria infection in house sparrows. Parasit. Vect. 9:232. doi: 10.1186/s13071-016-1512-1517

Martínez-de la Puente, J., Díez-Fernández, A., Soriguer, R., Rambozzi, L., Peano, A., Meneguz, P., et al. (2020). Are malaria-infected birds more attractive to mosquito vectors. Ardeola 68, 251-264. doi: 10.13157/arla.68.1.2021.fo1

Marzal, A., Bensch, S., Reviriego, M., Balbontin, J., and De Lope, F. (2008). Effects of malaria double infection in birds: one plus one is not two. J. Evol. Biol. 21, 979-987. doi: 10.1111/j.1420-9101.2008.01545.x

Medeiros, M. C. I., Anderson, T. K., Higashiguchi, J. M., Kitron, U. D., Walker, E. D., Brawn, J. D., et al. (2014). An inverse association between West Nile virus serostatus and avian malaria infection status. Parasit. Vect. 7:415. doi: 10.1186/1756-3305-7-415

Nguyen, P. L., Vantaux, A., Hien, D. F., Dabiré, K. R., Yameogo, B. K., Gouagna, L.-C., et al. (2017). No evidence for manipulation of Anopheles gambiae, An. coluzzii and An. arabiensis host preference by Plasmodium falciparum. Sci. Rep. 7:9415. doi: 10.1038/s41598-017-09821-x

Palinauskas, V., Martínez-de la Puente, J., Hernández-Soto, R. S., and Marzal, A. (2020). "Experimental Parasitology and Ecoimmunology: concepts and opportunities in avian Haemosporidian studies," Avian Malaria and Related Parasites in the Tropics, eds D. Santiago-Alarcon and A. Marzal (Cham: Springer).

Palinauskas, V., Valkiūnas, G., Bolshakov, C. V., and Bensch, S. (2011). Plasmodium relictum (lineage SGS1) and Plasmodium ashfordi (lineage GRW2): the effects of the co-infection on experimentally infected passerine birds. Exp. Parasitol. 127, 527-533. doi: 10.1016/j.exppara.2010.10.007

Palinauskas, V., Žiegytė, R., Iezhova, T. A., Ilgūnas, M., Bernotienè, R., and Valkiūnas, G. (2016). Description, molecular characterisation, diagnostics and life cycle of Plasmodium elongatum (lineage pERIRUB01), the virulent avian malaria parasite. Int. J. Parasitol. 46, 697-707. doi: 10.1016/j.ijpara.2016.05.005

Rivero, A., and Gandon, S. (2018). Evolutionary ecology of avian malaria: past to present. Trends Parasitol. 34, 712-726. doi: 10.1016/j.pt.2018.06.002

Robinson, A., Busula, A. O., Voets, M. A., Beshir, K. B., Caulfield, J. C., Powers, S. J., et al. (2018). Plasmodium-associated changes in human odor attract mosquitoes. Proc. Natl. Acad. Sci. U.S.A. 115, E4209-E4218. doi: 10.1073/pnas. 1721610115

Rochlin, I., Faraji, A., Healy, K., and Andreadis, T. G. (2019). West Nile virus mosquito vectors in North America. J. Med. Entomol. 56, 1475-1490. doi: 10.1093/jme/tjz146

Russell, C. B., and Hunter, F. F. (2005). Attraction of Culex pipiens/restuans (Diptera: Culicidae) mosquitoes to bird Uropygial gland odors at two elevations in the niagara region of Ontario. J. Med. Entomol. 42, 301-305. doi: 10.1093/ jmedent/42.3.301

Salibian, A., and Montalti, D. (2009). Physiological and biochemical aspects of the avian uropygial gland. Braz. J. Biol. 69, 437-446. doi: 10.1590/s151969842009000200029 
Santa-Ana, M., Khadem, M., and Capela, R. (2006). Natural infection of Culex theileri (Diptera: Culicidae) with Dirofilaria immitis (Nematoda: Filarioidea) on Madeira Island. Portugal J. Med. Entomol. 43, 104-106. doi: 10.1093/jmedent/ 43.1.104

Schaber, C. L., Katta, N., Bollinger, L. B., Mwale, M., Mlotha-Mitole, R., Trehan, I., et al. (2018). Breathprinting reveals malaria-associated biomarkers and mosquito attractants. J. Infect. Dis. 217, 1553-1560. doi: 10.1093/infdis/jiy072

Scott, T. W., Lorenz, L. H., and Edman, J. D. (1990). Effects of house sparrow age and arbovirus infection on attraction of mosquitoes. J. Med. Entomol. 27, 856-863. doi: 10.1093/jmedent/27.5.856

Simpson, J. E., Folsom-O’Keefe, C. M., Childs, J. E., Simons, L. E., Andreadis, T. G., and Diuk-Wasser, M. A. (2009). Avian host-selection by Culex pipiens in experimental trials. PLoS One 4:e7861. doi: 10.1371/journal.pone.0007 861

Smallegange, R. C., van Gemert, G. J., van de Vegte-Bolmer, M., Gezan, S., Takken, W., Sauerwein, R. W., et al. (2013). Malaria infected mosquitoes express enhanced attraction to human odor. PLoS One 8:e63602. doi: 10.1371/journal. pone.0063602

Spanoudis, C. G., Andreadis, S. S., Bray, D. P., Savopoulou-Soultani, M., and Ignell, R. (2020). Behavioural response of the house mosquitoes Culex quinquefasciatus and Culex pipiens molestus to avian odours and its reliance on carbon dioxide. Med. Vet. Entomol. 34, 129-137. doi: 10.1111/mve.12429

Takken, W., and Verhulst, N. O. (2013). Host preferences of blood-feeding mosquitoes. Annu. Rev. Entomol. 58, 433-453. doi: 10.1146/annurev-ento120811-153618

Valkiūnas, G. (2005). Avian Malaria Parasites and Other Haemosporidia, 1st Edn, Boca Raton, FL: CRC Press.

Valkiūnas, G., Bensch, S., Iezhova, T. A., Križanauskienë, A., Hellgren, O., and Bolshakov, C. V. (2006). Nested cytochrome b polymerase chain reaction diagnostics underestimate mixed infections of avian blood haemosporidian parasites: microscopy is still essential. J. Parasitol. 92, 418-422. doi: 10.1645/ ge-3547rn.1
Valkiūnas, G., Kazlauskienë, R., Bernotienë, R., Bukauskaitë, D., Palinauskas, V., and Iezhova, T. A. (2014). Haemoproteus infections (Haemosporida, Haemoproteidae) kill bird-biting mosquitoes. Parasitol. Res. 113, 1011-1018. doi: 10.1007/s00436-013-3733-4

Valkiūnas, G., Kazlauskienë, R., Bernotienë, R., Palinauskas, V., and Iezhova, T. A. (2013). Abortive long-lasting sporogony of two Haemoproteus species (Haemosporida, Haemoproteidae) in the mosquito Ochlerotatus cantans, with perspectives on haemosporidian vector research. Parasitol. Res. 112, 2159-2169. doi: 10.1007/s00436-013-3375-6

Vázquez, A., Ruiz, S., Herrero, L., Moreno, J., Molero, F., Magallanes, A., et al. (2011). West Nile and Usutu viruses in mosquitoes in Spain, 2008-2009. Am. J. Trop. Med. Hyg. 85, 178-181. doi: 10.4269/ajtmh.2011.11-0042

Vogels, C. B. F., Fros, J. J., Pijlman, G. P., van Loon, J. J. A., Gort, G., and Koenraadt, C. J. M. (2017). Virus interferes with host-seeking behaviour of mosquito. J. Exp. Biol. 220, 3598-3603. doi: 10.1242/jeb.164186

Yan, J., Broggi, J., Martínez-de la Puente, J., Gutiérrez-López, R., Gangoso, L., Soriguer, R., et al. (2018a). Does bird metabolic rate influence mosquito feeding preference? Parasit. Vector. 11:110. doi: 10.1186/s13071-018-2708-9

Yan, J., Martínez-de la Puente, J., Gangoso, L., Gutiérrez-López, R., Soriguer, R., and Figuerola, J. (2018b). Avian malaria infection intensity influences mosquito feeding patterns. Int. J. Parasitol. 48, 257-264. doi: 10.1016/j.ijpara.2017.09.005

Conflict of Interest: The authors declare that the research was conducted in the absence of any commercial or financial relationships that could be construed as a potential conflict of interest.

Copyright (C) 2020 Santiago-Alarcon and Ferreira. This is an open-access article distributed under the terms of the Creative Commons Attribution License (CC BY). The use, distribution or reproduction in other forums is permitted, provided the original author(s) and the copyright owner(s) are credited and that the original publication in this journal is cited, in accordance with accepted academic practice. No use, distribution or reproduction is permitted which does not comply with these terms. 